Relationships, Sexual assault and The Law. Interestingly, sites with more innovative features had less clarity, and vice versa. Examples of innovative features include image galleries/videos/films, newsfeeds, voting tools, online feedback forms, contraception tools, clinic finders, online booking and risk assessment tools.

Discussion/Conclusions Populating the website with more innovative features appears to reduce clarity. Are they mutually exclusive? To answer that question, user feedback should be sought and web site analytics used to determine if features have real utility or are just superfluous.

\section{P108 "DON'T FORGET THE CHILDREN", USING ELECTRONIC PATIENT RECORDS AS A TOOL TO ACHIEVE HIV TESTING OF CHILDREN OF HIV POSITIVE PARENTS}

doi:10.1136/sextrans-2012-050601c.108

R Naidoo, ${ }^{*}$ B Williams, S Murphy, M Le Prevost, J McSorley, G Brook, J Alagaratnam. Northwest London Hospital NHS Trust, Harrow, UK

Background The HIV status of children of HIV positive adults in the UK should be known as a matter of urgency. Implementing robust systems to identify and then test this vulnerable group of children remains a challenge.

Aims We present a single service experience of using Electronic Patient Records (EPR) to help identify such children.

Methods A clinical proforma to record the HIV status of all children of all HIV positive adults attending this service was developed on the EPR system in early 2010. Using the EPR Lilie database the records of all adult patients attending the service in 2010 and 2011 were searched for details of the HIV status of their children under 18 years. Results There were 389 HIV positive patients and 306 children identified. 278 of these children live in the UK. Of these, $242(87 \%)$ had a verified HIV status and $36(13 \%)$ an unverified HIV status. Of the 36 with unverified status, 12 (33\%) were said to be HIV tested (negative) but this was unconfirmed, 9 (25\%) had an HIV positive father and a mother who was said to be HIV negative but this was unconfirmed. 7 (19\%) had been referred to paediatrics for testing and $8(22 \%)$ children were untested for mixed reasons.

Discussion Verifying the HIV status of the children of HIV positive parents is a complex, multidisciplinary exercise requiring excellent documentation and cooperation between different teams. The first crucial step is identifying the at risk children. The proforma in the patient records acted as a prompt to the clinician to obtain this information from the patient. Information was then easily identifiable in the EPR. The results demonstrate the strength of the EPR system. All proformas were completed showing that of our cohort of children living in the UK $87 \%$ have verified HIV status and in $13 \%$ there is enough information to pursue HIV testing or verification.

\section{P109 INTRODUCTION OF AN ELECTRONIC PATIENT PROFORMER CONTRIBUTES TO AN INCREASE IN UPTAKE OF HIV TESTING}

doi:10.1136/sextrans-2012-050601c.109

S Bhatt, E Clarke, ${ }^{*}$ K Roseaman, R Patel, S Samraj. Royal South Hants Hospital, Southampton, UK

Background The BASHH MEDFASH Standards for the Management of STIs 2010 recommend that $100 \%$ of GUM patients should be offered an HIV test with a minimum uptake of $60 \%$ at their first STI screen.

Aim To assess whether the introduction of an electronic patient proformer resulted in an improvement in the uptake of HIV testing in a level 3 GUM service, and whether there was scope for further improvement.
Methods Retrospective case note review of new and rebook (patients who had not attended in the past 3 months) patients attending a level 3 GUM service in October 2007 (when a paper patient record was kept) and October 2010 (following introduction of an electronic patient proformer with a prompt for HIV test offered). Data on uptake of HIV testing was collected, and further data on the reasons for not being tested for HIV was collected on patients seen in October 2010.

Results 772 new or rebook patients were seen in October 2007, of whom $562(72.8 \%)$ accepted HIV testing. 1141 new or rebook patients were seen in October 2010 of whom 891 (78.1\%) accepted HIV testing, with a p value (calculated using Fisher's Exact test) of 0.009. Of $250(21.9 \%)$ patients not tested for HIV in October 2010, $41.6 \%$ were not tested for clinical reasons, such as the patient having had a recent HIV test or being known HIV positive. $35.2 \%$ of patients not tested for HIV declined the test. Evidence of suboptimal management was also found, with $6.8 \%$ of patients not tested as they were within the window period, and $6 \%$ not tested due to needle phobia.

Conclusion The introduction of the electronic patient proformer with a prompt for HIV testing has improved uptake. There is further room for improvement including offering POCT to needle phobic patients, and testing those within the window period prior to follow-up testing. Adding a mandatory field to the patient proformer with reasons for declining may allow improved understanding of reasons for declining, which may then modify future practice.

\section{P110 DEVELOPMENT OF A WEB-BASED PARTNER NOTIFICATION NETWORK LINKING GENERAL PRACTICE, CASH SERVICES AND COMMUNITY PHARMACY WITH SPECIALIST GUM SERVICES}

doi:10.1136/sextrans-2012-050601c.110

${ }^{1} \mathrm{~L}$ Sutcliffe, ${ }^{*}{ }^{2} \mathrm{M}$ Symonds, ${ }^{3} \mathrm{~T}$ Roberts, ${ }^{4} \mathrm{C}$ Mercer, ${ }^{1} \mathrm{C}$ Estcourt. ${ }^{1}$ Barts \& The London School of Medicine \& Dentistry, London, UK; ${ }^{2}$ Barts \& The London NHS Trust, London, UK; ${ }^{3}$ Health Economics Unit, University of Birmingham, Edgbaston, UK; ${ }^{4}$ Centre for Sexual Health \& HIV Research, UCL, London, UK

Background Partner notification in primary care is problematic and of limited effectiveness despite enthusiasm from primary care providers to engage with sexually transmitted infection (STI) management. Often it relies on the clinician informing the patient to advise his/her sex partners to attend a sexual health clinic for investigations and treatment despite evidence suggesting that only around half of sex partners informed in this way receive treatment. Many of those referred on to a sexual health clinic by a GP fail to attend and follow-up is infrequent.

Aim To develop a robust, web-based tool for managing partner notification for patients diagnosed with bacterial STIs in primary care with broad geographical and service applicability for use in our Accelerated Partner Therapy randomised control trial in primary care. Method We applied a user-centered design approach to develop a web-based partner notification tool linking different types of community sexual health providers with the specialist GU service. The approach included a pre-design phase using discussions with primary care health professionals, and sexual health clinic health advisers, to determine their needs, current activity and work habits, to ensure that the web-based tool would be successfully integrated in their daily work practice. The web-tool was designed to our specifications by a bespoke software company.

Results and Discussion We have designed a secure, simple to use web tool that allows users in a variety of settings to easily initiate, manage and monitor partner notification. A major advantage of this platform compared to other visualisation tools is its web-based format, which requires no software installation or data downloads. 
Data are collected in real time, and all partner notification outcomes are recorded. The platform is extendable to facilitate centralised partner notification within sexual health clinical networks across many different heath care providers.

\section{P111 A USER CENTRED APPROACH TO THE DESIGN OF POINT- OF-CARE AND SELF-TEST MOBILE PHONE DIAGNOSTICS FOR SEXUALLY TRANSMITTED INFECTIONS (STIS)}

\author{
doi:10.1136/sextrans-2012-050601c.111
}

${ }^{1} \mathrm{G}$ Voula, ${ }^{*}{ }^{1} \mathrm{~W}$ Balachandran, ${ }^{2} \mathrm{C}$ Lowndes, ${ }^{2} \mathrm{R}$ Howell-Jones, ${ }^{3} \mathrm{C}$ R Aicken, ${ }^{3} \mathrm{C} H$ Mercer, ${ }^{4} \mathrm{~L}$ Sutcliffe, ${ }^{3} \mathrm{P}$ Sonnenberg, ${ }^{5} \mathrm{M}$ Jackson, ${ }^{4} \mathrm{C}$ S Estcourt, ${ }^{6} \mathrm{~S}$ T Sadiq, ${ }^{1} \mathrm{~K}$ Hone. ${ }^{1}$ Brunel University, Middlesex, UK; ${ }^{2}$ Health Protection Agency; ${ }^{3}$ University College London, London, UK; ${ }^{4}$ Queen Mary University of London; ${ }^{5}$ xscient; ${ }^{6}$ St Georges University of London

Background Control of STIs in vulnerable populations is difficult because of inadequate access to prompt diagnosis and treatment and may be enhanced by better community based STI surveillance. The high rates of mobile and smart phone use in these populations and the development of novel personalised diagnostic technologies which theoretically enable highly accurate self-testing diagnostics for STIs that can be electronically linked to clinical care pathways and STI data capture, may help address these challenges. A major challenge for these systems is the development of clinically relevant and acceptable Human Technology Interfaces (HTIs) for Mobile-Web Applications. Objectives To describe a methodological approach to develop a mobile phone/web-based management system that can link rapid self-test diagnostics to clinical care pathways and community based surveillance.

Methods We adopt a user centred approach to the development of a HTI for self-managing STI diagnosis with initial exploratory pilot studies to gather functional, user and clinical requirements of the HTI. Iterative development of functional prototypes exploring design possibilities and technology features is followed by the formative evaluation of interface design alternatives through qualitative and controlled usability studies with target users and existing surveillance capture mechanisms.

Results A user centred, multi-disciplinary approach allows for successful capture and integration of social science methods to inform functional requirements for an effective and user acceptable mobile and web-based self-managing STI diagnostics and surveillance system.

Discussion Future testing in simple and complex, community based studies are needed to primarily assess safety and then effectiveness for improving access to care, enhanced partner notification and development of community based capture of surveillance data.

\section{P112 TEXTING OR TALKING? WHICH WORKS BETTER TO REDUCE HAZARDOUS DRINKING BY SEXUAL HEALTH CLINIC ATTENDEES?}

doi:10.1136/sextrans-2012-050601c.112

\section{S Baguley.* NHS Grampian}

Background Hazardous drinking is associated with an increased risk of catching an STI, getting pregnant unintentionally, being sexually assaulted and sexually assaulting someone else. An Alcohol Brief Intervention ( $\mathrm{ABI}$ ), a type of motivational interview, has been shown to reduce hazardous drinking in men over the age of 25 . There is an paucity of evidence of the benefit in younger people or in women. Texting has been shown to be useful in supporting smoking cessation, weight reduction and diabetes control. There have been no studies of its potential value in reducing hazardous drinking or its consequences.
Aim To compare the effect of texting vs an ABI on hazardous drinking and associated sexual illhealth amongst attendees at an urban sexual health clinic.

Methods Potential subjects are being recruited from a city sexual health clinic. Initial screening is done using the Alcohol Use Disorder Identification Test - a self-completed questionnaire marked out of 40. Consenting subjects are then randomised to texting (12 texts over 4 months), an ABI or a leaflet and then followed up by phone at 4 months. At follow-up, subjects are rescreened for hazardous drinking and asked about incident STIs, regretted sex, sexual assault, use of emergency contraception and for their evaluation of the intervention method.

Results To end of January 2012, 6279 attendees have been screened of whom 2554 were hazardous drinkers and 799 have consented to take part in the study. (The target sample size is 1090). By the time of the conference, all subjects will have been recruited and most results will be available.

Conclusion This is the first RCT of texting for hazardous drinking and should inform national policy in this area.

\section{P113 PRIMARY HEALTH PROVIDERS' ADHERENCE TO NATIONAL CHLAMYDIA GUIDELINES}

doi:10.1136/sextrans-2012-050601c.113

\author{
${ }^{1} \mathrm{~J}$ M Morgan, ${ }^{* 2} \mathrm{~L}$ A Mills, ${ }^{1} \mathrm{~A} \mathrm{~J}$ Bell. ${ }^{1}$ Waikato Hospital; ${ }^{2}$ University of Otago
}

Aim To re-audit district-wide management of genital chlamydia infection following implementation of $\mathrm{NZ}$ national chlamydia guidelines.

Methods All healthcare settings in a single NZ health district that diagnosed 20 or more cases of chlamydia during September 2010-2011 were invited to participate and were asked to complete an audit proforma for 20 consecutive cases. Sexual Health clinic cases were excluded. Collated results were compared to findings of a similar audit of 394 cases diagnosed during 2008, which was undertaken before guideline implementation in 2009.

Results 19 of 22 eligible sites participated, providing data for 424 cases; of these, 302 (71.2\%) were managed in General Practice, with the remainder from a range of settings including Family Planning, student health services, and A\&E. 405 of 424 (95.5\%) cases in 2010-2011 had documented treatment compared to 354 of 394 $(89.8 \%)$ in 2008. Notifying current sexual partners was noted in 314 cases $(74 \%)$, compared to $58.1 \%$ in 2008.126 of 424 (29.7\%) of cases were noted to have informed at least $50 \%$ of their sexual partners compared to $17.8 \%$ in 2008 .

Conclusion Improved case management and partner notification may reflect better documentation than in the initial audit but more appropriate choice of diagnostic sampling, more testing for other STIs and more appropriate dosage and duration of prescribed antibiotics supports that primary care chlamydia case management in this district has improved following local implementation of national guidelines. More work is needed to enhance documented partner management outcomes.

\section{Improving clinical practice and service delivery}

\begin{tabular}{l|l}
\hline P114 IS THERE A ROLE FOR PRIMARY CARE CLINICIANS IN \\
PROVIDING SHARED CARE IN HIV TREATMENT? A \\
SYSTEMATIC LITERATURE REVIEW
\end{tabular}

doi:10.1136/sextrans-2012-050601c.114

${ }^{1}$ W Wong, ${ }^{1} \mathrm{C}$ W Luk, ${ }^{2} \mathrm{M}$ R Kidd. ${ }^{1}$ The University of Hong Kong; ${ }^{2}$ Faculty of Health Sciences, Flinders University

Background Despite HIV being increasingly considered as a chronic illness, there is as yet no consensus about how primary care should 